University of Nebraska - Lincoln

DigitalCommons@University of Nebraska - Lincoln

$9-15-1998$

\title{
Wave-vector-dependent exchange splitting in a local moment system
}

\author{
C. Waldfried \\ University of Nebraska-Lincoln \\ T. McAvoy \\ University of Nebraska-Lincoln \\ D. Welipitiya \\ University of Nebraska-Lincoln \\ Takashi Komesu \\ University of Nebraska-Lincoln, tkomesu2@unl.edu \\ Peter A. Dowben \\ University of Nebraska-Lincoln, pdowben@unl.edu \\ See next page for additional authors
}

Follow this and additional works at: https://digitalcommons.unl.edu/physicsdowben

Part of the Physics Commons

Waldfried, C.; McAvoy, T.; Welipitiya, D.; Komesu, Takashi; Dowben, Peter A.; and Vescovo, Elio, "Wavevector-dependent exchange splitting in a local moment system" (1998). Peter Dowben Publications. 71. https://digitalcommons.unl.edu/physicsdowben/71

This Article is brought to you for free and open access by the Research Papers in Physics and Astronomy at DigitalCommons@University of Nebraska - Lincoln. It has been accepted for inclusion in Peter Dowben Publications by an authorized administrator of DigitalCommons@University of Nebraska - Lincoln. 


\section{Authors}

C. Waldfried, T. McAvoy, D. Welipitiya, Takashi Komesu, Peter A. Dowben, and Elio Vescovo 


\title{
Wave-vector-dependent exchange splitting in a local moment system
}

\author{
C. Waldfried, T. McAvoy, D. Welipitiya, Takashi Komesu, and P. A. Dowben* \\ Department of Physics and Astronomy and the Center for Materials Research and Analysis, Behlen Laboratory of Physics, \\ University of Nebraska-Lincoln, Lincoln, Nebraska 68588-0111 \\ E. Vescovo \\ National Synchrotron Light Source, Brookhaven National Laboratory, Upton, New York 11973
}

(Received 9 April 1998)

\begin{abstract}
The electronic structure of strained thin films of Gd has been studied with spin- and angle-resolved photoemission and spin-polarized inverse photoemission. The spin-dependent electronic structure is dominated by a very distinct $k$ dependence of the exchange splitting. The surface magnetic structure is observed to be different from that of the bulk, as indicated by the different electronic structure and a much higher surface Curie temperature. The $4 \%$ strain within the Gd films results in an enhanced Curie temperature.
\end{abstract}

[S0163-1829(98)06435-2]

\section{INTRODUCTION}

It has been postulated that the magnetic coupling ${ }^{1-4}$ and exchange splitting ${ }^{5-13}$ are wave-vector dependent, although no direct experimental evidence has been provided to date to confirm this proposal in an elemental system. Indirect affirmation for $k$-dependent exchange splitting has been provided for $\mathrm{Gd}(0001),{ }^{12-14} \mathrm{Ni},{ }^{15,16} \mathrm{Fe},{ }^{17,18}$ and Co. ${ }^{19}$ Gadolinium is a ferromagnet where spin-spin coupling (dipole-dipole interactions) is generally considered to be strong, while spin-orbit interactions are expected to be weak, because of the halffilled $4 f$ shell. Crystal-field effects may couple the dipole to the lattice, leading to some crystalline anisotropy. Nonetheless, because of the strong dipole coupling, gadolinium is less likely than transition-metal ferromagnetic systems to exhibit a wave-vector-dependent exchange splitting. Many have come to expect to observe a pronounced wave-vector dependence of the exchange splitting primarily in compound systems where the lattice contains atoms with both small and large moments in an ordered array; such is the case of cobalt with chemisorbed oxygen ${ }^{20}$ or the rare-earth pnictides ${ }^{21}$ but not in local moment elemental systems. In reality, this desire for simplicity really has no foundation in band structure.

While a number of studies ${ }^{22-24}$ find a largely Stoner-like temperature dependence of the exchange splitting for $\mathrm{Gd}(0001)$, and one such study ${ }^{22}$ suggested that the exchange splitting was wave-vector independent, there is no a priori basis for either conclusion. Finite temperature band-structure calculations ${ }^{5}$ suggest that the exchange splitting in gadolinium is, in fact, wave-vector dependent. Further, gadolinium is expected to be an example of wave-vectordependent coupling. ${ }^{2-4}$

The temperature dependence of the gadolinium band structure is dominated by the interplay between Stoner-like ferromagnetism where the exchange splitting collapses to zero at $T_{C}$ and spin-mixing behavior where there are four subbands whose populations tend towards equal weight at $T_{C}$ but the binding energies do not shift with temperature. $5,12,25,26$ The magnetic structure of gadolinium is strongly influenced by the intra-atomic $4 f-(5 d, 6 s)$ wave- function overlap and $5 d, 6 s$ itinerancy. ${ }^{14}$ Expansive strain within the hexagonal closed-packed system substantially alters the electronic and magnetic valence-band structure. ${ }^{27}$ The strain should result in increased electron localization of the itinerant $5 d, 6 s$ valence electrons ${ }^{14,27,28}$ and possibly increase the Curie temperature. ${ }^{29,30}$ With this in mind, we studied the wave-vector dependence of the spin-resolved band structure of strained thin films of Gd (Ref. 27) with spin- and angle-resolved photoelectron spectroscopy.

\section{EXPERIMENT}

Strained thin films of gadolinium with an increased lattice constant of approximately $4 \%$ as compared to $\mathrm{Gd}(0001)$, and a well ordered hexagonal surface unit cell were obtained by growing $\mathrm{Gd}$ on the corrugated surface of $\mathrm{Mo}(112) .{ }^{27}$ The wave-vector-dependent electronic structure of Gd films of 15 to 40 monolayers (ML) thickness was studied in a UHV system at the new U5UA undulator beamline at the National Synchrotron Light Source (NSLS) at the Brookhaven National Laboratory, ${ }^{31}$ using a spin- and angle-resolved photoemission analyzer, as described in detail elsewhere. ${ }^{32}$ The Gd films were magnetized in-plane along the substrate corrugation lines and spin-polarized photoemission spectra were acquired in remanence using photons of $35.2 \mathrm{eV}$, incident at an angle of $65^{\circ}$ relative to the surface normal. The combined energy and angular resolution were better than $0.15 \mathrm{eV}$ and $\pm 1^{\circ}$, respectively. The surface and bulk character of the $\mathrm{Gd}$ bands has been determined from chemisorption studies and photon energy dependence, while the symmetry of the bands has been ascertained from the light polarization dependence studies as described in detail elsewhere. ${ }^{27}$

The spin-polarized inverse photoemission experiments were undertaken with a transversely polarized spin electron gun based upon the Ciccacci design. ${ }^{33}$ The spin electron gun was designed in a compact form on a separate chamber equipped with an iodine based Geiger-Müller isochromat photo detector. ${ }^{34,35}$ The spin-polarized electrons were emitted from a GaAs photocathode into a spin-rotator ${ }^{33,36,37}$ and subsequently to the electron optics. ${ }^{33,36-38}$ The direction of 


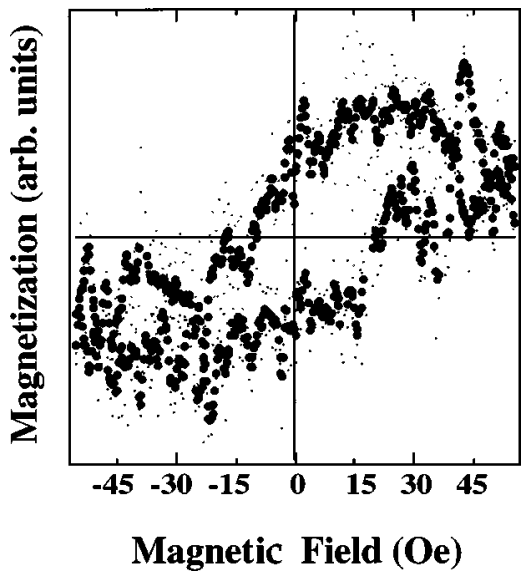

FIG. 1. The in-plane hysteresis loop of a 10-ML-thick film of strained gadolinium grown on $\mathrm{Mo}(112)$. The magneto-optic Kerr effect hysteresis loop was taken at $130 \mathrm{~K}$.

electron polarization is in the plane of the sample for all incidence angles, as is the applied field, and spectra were obtained at remanence. The GaAs photocathode was mounted on a sample transfer arm separated from the spectroscopy chamber by a gate valve so that cleaning of both the $\mathrm{Mo}(112)$ substrate and the GaAs photocathode could be accomplished without cross contamination.

Moke studies were undertaken to obtain the hysteresis loop of the in-plane magnetization. A SpetraPhysics 117a 1.0 $\mathrm{mW}$ He-Ne laser $(\lambda=632.8 \mathrm{~nm})$, chopped at a frequency of $1 \mathrm{kHz}$ and linearly polarized, was used. As seen in Fig. 1, the coercivity of the strained $\mathrm{Gd}(0001)$ films (10 ML in this example) is about $30 \mathrm{Oe}$. Both the spin-polarized photoemission and spin-polarized inverse photoemission are undertaken in remanence, and since the applied pulsing fields are in excess of $300 \mathrm{Oe}$, the films are clearly saturated after each pulse. Remanence is seen to be quite high (Fig. 1). Instrumental asymmetry in the electron spectroscopies has been removed by alternating the direction of the field after each sweep of electron energy analyzer (spin-polarized photoemission) or the electron gun energy (spin-polarized inverse photoemission) and summing appropriately to form the spectra.

\section{THE INFLUENCE OF STRAIN ON THE SPIN- POLARIZED BAND STRUCTURE OF Gd(0001)}

Strained thin films of gadolinium with an increased lattice constant of about $4 \%$ as compared to $\operatorname{Gd}(0001)$ have been obtained by growing $\mathrm{Gd}$ on the corrugated surface of Mo(112). The growth, structure, and spin-integrated electronic structure of these strained gadolinium films have been described at great length. ${ }^{27}$ Ultrathin $(3<d<10 \mathrm{ML})$ and thin $(d>10 \mathrm{ML})$ films of Gd order in well defined rectangular and hexagonal surface unit cells, which resemble strained $\operatorname{Gd}(10 \overline{1} 2)$ and strained $\operatorname{Gd}(0001)$, respectively. ${ }^{27}$

The influence of strain on the spin-polarized electronic structure of Gd is illustrated in Fig. 2, where the combination of normal-emission spin-polarized photoemission spectra and normal incidence $(\bar{\Gamma})$ spin-polarized inverse photoemission were taken for strained $[\mathrm{Gd}$ on $\mathrm{Mo}(112)]$ and unstrained $[\mathrm{Gd}$ on $\mathrm{W}(110)] \mathrm{Gd}(0001)$ at $k_{\|}=0$ or $\bar{\Gamma}$. The "unstrained"

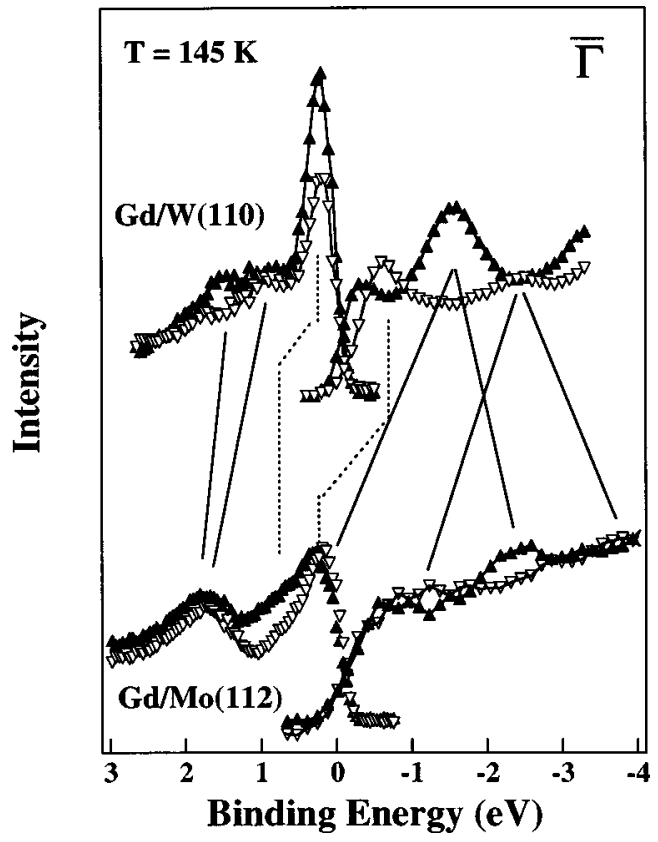

FIG. 2. Spin-polarized photoemission spectra (left) and spinpolarized inverse photoemission (right) for $\mathrm{Gd}(0001)$ on $\mathrm{W}(110)$ (unstrained) and $\mathrm{Gd}(0001)$ on $\mathrm{Mo}(112)$ (strained) at $k_{\|}=0$ or $\bar{\Gamma}$ and approximately $145 \mathrm{~K}$. The lines schematically indicate the bindingenergy shifts of the surface (dashed) and bulk (solid) spin subbands as a function of increased expansive strain. The spin-polarized inverse photoemission spectra for unstrained $\mathrm{Gd}(0001)$ grown on W(110) are taken from Ref. 39.

$\operatorname{Gd}(0001)$ spin-polarized inverse photoemission data in Fig. 2 were adopted from Donath and co-workers. ${ }^{39}$ Both the occupied and unoccupied bands of the strained $\operatorname{Gd}(0001)$ films grown on $\mathrm{Mo}(112)$ are distinct from that of the relaxed films grown on $\mathrm{W}(110)$.

The unstrained $\mathrm{Gd}(0001)$ valence band at the Brillouinzone center $(\bar{\Gamma})$ is characterized by Stoner-like exchange split $5 d$ bulk bands ${ }^{22,25}$ at binding energies of approximately 1.5 (majority) and $0.8 \mathrm{eV}$ (minority) and two sets of spinmajority and -minority subbands of the surface on either side of the Fermi level. ${ }^{23,25,39-41}$ There is also a pair of exchange split unoccupied bulk bands observed well above the Fermi level. ${ }^{39}$

In the strained Gd films, the $5 d, 6 s$ occupied bulk spinmajority and -minority subbands are found at approximately $1.8 \mathrm{eV}$ binding energy at $\bar{\Gamma}$, with negligible Stoner-like exchange splitting. These bulk bands change symmetry from

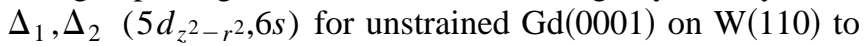
$\Delta_{5}, \Delta_{6}$ at $\bar{\Gamma}$ with the $4 \%$ expansive strain on $\operatorname{Mo}(112){ }^{27}$ There are other bands, with at least some bulk character, both near and well above the Fermi level, as indicated in Fig. 2.

The narrow surface state near the Fermi level of the unstrained $\mathrm{Gd}(0001)$ is also shifted towards higher binding energy and appears substantially broadened with expansive strain. For "unstrained" $\mathrm{Gd}(001)$ grown on $\mathrm{W}(110)$, the oc-

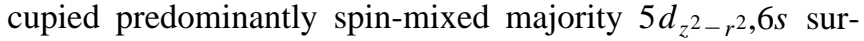
face state is located at approximately $0.1 \mathrm{eV}$ below $E_{F}$ (Figs. 2 and 3) and the unoccupied minority counter part of the surface state at approximately $0.3 \mathrm{eV}$ above the Fermi level ${ }^{39}$ (Fig. 2), though the binding energy of the various compo- 

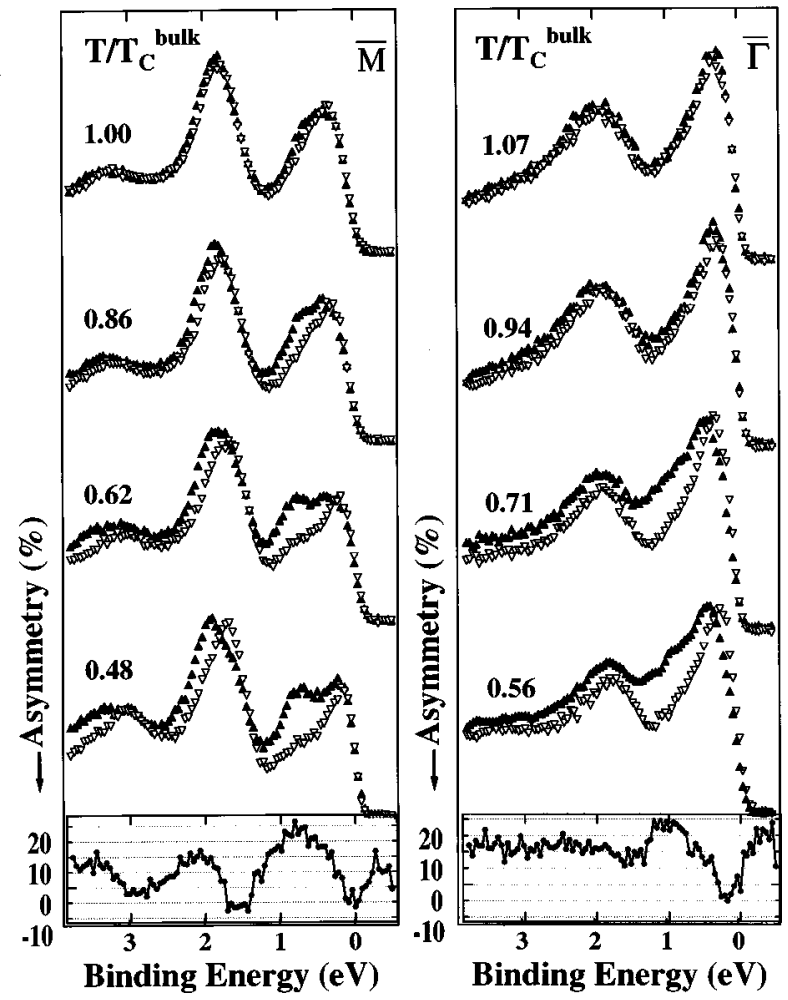

FIG. 3. The temperature dependence of the valence band of strained Gd near the zone edge $\bar{M}$ (left panel) and at the zone center $\Gamma$ (right panel). Spin-majority and spin-minority components are indicated by $(\boldsymbol{\Lambda})$ and $(\nabla)$, respectively. The temperatures are indicated as a function of the corresponding bulk Curie temperature. The spin asymmetries for the low-temperature spectra are displayed in the bottom of each panel. The spectra at $\bar{M}$ were acquired for a Gd film of 40-ML thickness with a corresponding bulk Curie temperature of approximately $340 \mathrm{~K}$, while the spectra at normal emission are taken for a 16-ML Gd film with a bulk Curie temperature of approximately $270 \mathrm{~K}$.

nents of the surface state is dependent on temperature well away from $T_{C} \cdot{ }^{23,39,42}$ In the valence band of the strained $\mathrm{Gd}(0001)$ films grown on $\mathrm{Mo}(112)$, there are three features near the Fermi level ( $0-1 \mathrm{eV}$ binding energy). Two features, the spin-majority state at $0.7 \mathrm{eV}$ below $E_{F}$ and the spinminority state at $0.2 \mathrm{eV}$ binding energy (though this band may possibly cross over the Fermi level) are the exchange split surface bands. The surface character of these two states has been verified by their sensitivity to small amounts of adsorbates and their two-dimensionality of state. Both surface-sensitive features do not disperse with changing perpendicular momentum ${ }^{27}$ and are therefore confined to the two-dimensional plane at the surface. Expansive strain within the Gd films induces a "downward" shift of the unoccupied surface state across the Fermi level but without the symmetry change that occurs with strain for the bulk bands.

There is a third feature in the valence-band region near $E_{F}$ for strained $\mathrm{Gd}(0001)$ grown on $\mathrm{Mo}(112)$. This is an additional bulk band of majority character, located at approximately $0.4 \mathrm{eV}$ binding energy at $T / T_{C}^{B}<0.7$. This bulk band may also actually trail across the Fermi level to the unoccupied side and this is certainly suggested by the spin-polarized inverse photoemission spectra (Fig. 2), though it is by no means conclusive. (Such a straddling across the Fermi level of this spin-majority bulk band would act to cancel some of the net polarization of the spin-minority surface state that also may cross the Fermi level, thus leading to little net polarization in our spin-polarized inverse spectra, as observed.) The spin-majority bulk band at $0.4 \mathrm{eV}$ binding energy of the strained Gd film continues to have an unoccupied counterpart, and the combined spin-polarized photoemission and spin-polarized inverse photoemission indicate an exchange splitting of this bulk band of about 1 to $1.5 \mathrm{eV}$ at $\bar{\Gamma}$. The "corresponding" bulk bands in "unstrained" $\mathrm{Gd}(0001)$ are located approximately $1.5 \mathrm{eV}$ above the Fermi level, ${ }^{39}$ at the zone center, and cross the Fermi level about halfway across the zone in unstrained Gd. This shift, with expansive strain, in the unoccupied bulk band binding energies at $\bar{\Gamma}$ is also illustrated in Fig. 2. The expansive strain induced the binding-energy shift of some of the bulk bands [near the Fermi level in strained $\mathrm{Gd}(0001)]$ and also appears to be accompanied by a symmetry change from $\Delta_{5}, \Delta_{6}$ (Ref. 43) to $\Delta_{1}, \Delta_{2}\left(5 d_{z^{2}-r^{2}}, 6 s\right)$ at $\bar{\Gamma} .{ }^{27}$ Accompanying the expansive strain, there must be a substantial change in the band structure, elsewhere in the Brillouin zone, to both preserve charge neutrality and correctly populate bands.

The shift to higher binding energies of the Gd surface states (majority and minority) under the influence of expansive strain is in agreement ${ }^{44}$ with the strain-induced shifts of the surface-state binding energy for $\operatorname{Ag}(111) .{ }^{45}$ There, compressive strain results in the upward shift of the sharp $\operatorname{Ag}(111)$ surface state across the Fermi level, where it is cut off by the Fermi function and undetectable with photoemission. In both example $[\mathrm{Gd}(0001)$ and $\mathrm{Ag}(111)]$, the strainedinduced shift of the surface state(s) may be explained by the phase accumulation model. ${ }^{46}$

One profound consequence of the expansive strain is the change in the surface and bulk Curie temperatures. Using the surface state and bulk band exchange splitting and polarization we have been able to estimate the surface and bulk Curie temperatures. The thicker strained gadolinium (0001) films [approximately $40 \mathrm{ML}$ of $\mathrm{Gd}$ on $\mathrm{Mo}(112)$ ] exhibit an enhanced surface Curie temperature of $370 \pm 25 \mathrm{~K}$ and bulk Curie temperatures of $340 \pm 20 \mathrm{~K}^{47}$ These values are much higher than the expected values for unstrained gadolinium (0001) of $T_{C}^{B}=293 \mathrm{~K}$ and a $T_{C}^{S}$ ranging between $310 \mathrm{~K}$ (Ref. 48) and $350 \mathrm{~K}^{49}$ In fact, the strongly enhanced Curie temperatures for expansively strained $\mathrm{Gd}(0001)$ are all the more surprising in view of the fact that an enhanced surface Curie temperature for the thinner films of $\mathrm{Gd}(0001)$ grown on $\mathrm{W}(110)$ has been called into question. ${ }^{23,39,50}$ Nonetheless, the change in the critical temperatures with expansive strain is consistent ${ }^{51}$ with the decrease of the Curie temperature in gadolinium with pressure. ${ }^{52}$

\section{WAVE-VECTOR-DEPENDENT EXCHANGE SPLITTING}

The spin-resolved temperature-dependent electronic structure of the valence band of strained $\operatorname{Gd}(0001)$ is illustrated in Fig. 3, which shows valence-band spectra acquired at two distinct electron wave vectors - the surface Brillouin zone center, $\bar{\Gamma}\left(k_{\|}=0\right)$ (right), and near the zone edge $\bar{M}\left(k_{\|}\right.$ 

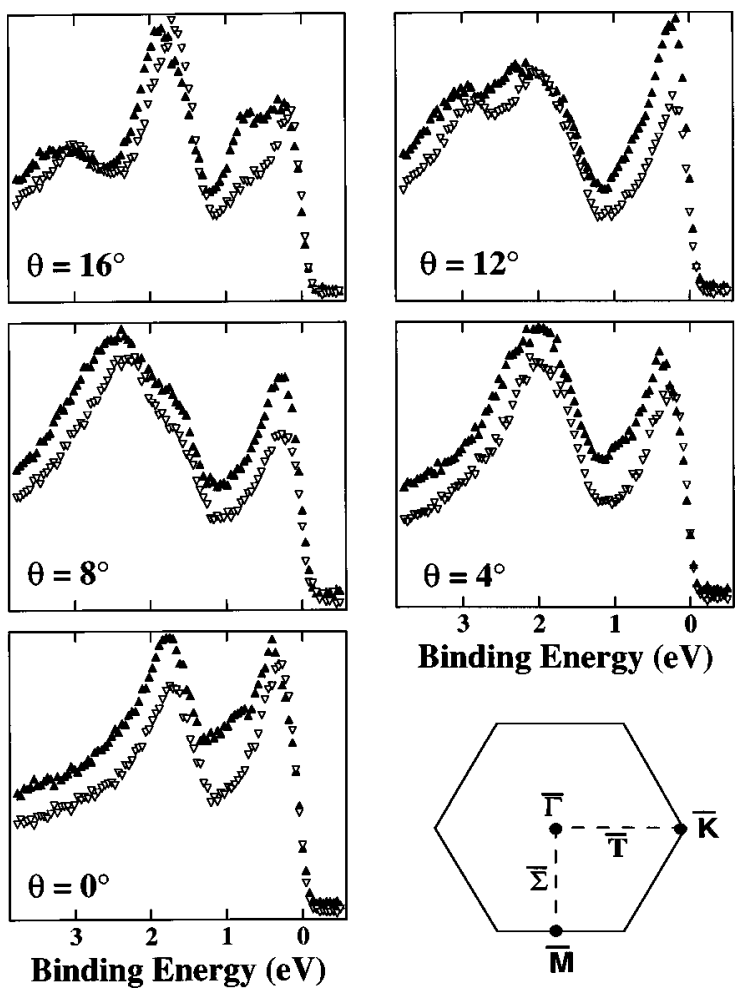

FIG. 4. Spin-polarized emission angle-dependent valence-band photoemission spectra for various points along the $\overline{\Gamma \Sigma M}$ highsymmetry line. The spectra were acquired for a 40-ML-thick strained Gd film at approximately $145 \mathrm{~K}$. Spin-majority and spinminority components are indicated by $(\boldsymbol{\Lambda})$ and $(\nabla)$, respectively. The bottom right panel displays the hexagonal surface Brillouin zone of strained Gd.

$=0.96 \AA^{-1}$ ) (left). There are compelling differences in the spin-resolved spectra for the two high symmetry points $\Gamma$ and $\bar{M}$.

For $40 \mathrm{ML}$ of strained $\mathrm{Gd}(0001)$ grown on $\mathrm{Mo}(112)$, the Gd $5 d$ bulk bands, at $1.8 \mathrm{eV}$ below $E_{F}$ at the zone center $(\bar{\Gamma})$, are characterized by spin-majority and spin-minority components with very similar binding energies and a spin asymmetry of approximately $12 \%$ for $T / T_{C}^{B}=0.56$. This spin asymmetry is comparable to the background polarization (2.5-4.0 eV binding energy) as seen in the plotted valenceband spin polarization on the bottom of Fig. 3. The uniform polarization in the region of $2 \mathrm{eV}$ binding energy at $\Gamma$ and the negligible difference in binding energy between the two spin components of the bulk band are indicative of a band with very little exchange splitting and little spin-mixing behavior. ${ }^{10,12}$ It is worth noting again that while the occupied bulk bands at $\bar{\Gamma}$ at $1.8 \mathrm{eV}$ binding energy exhibit no more ferromagnetic behavior than the background, the bulk bands near the Fermi level exhibit substantial ferromagnetic behavior and an exchange splitting of $1-1.5 \mathrm{eV}$ at $\Gamma$ (Fig. 2).

In contrast, near $\bar{M}$ the two nondegenerate $\mathrm{Gd} 5 d_{x z, y z}$ or $5 d_{x^{2}-y^{2}}$ occupied bulk bands ${ }^{27}$ at binding energies of approximately 1.8 and $3.0 \mathrm{eV}$ show a clear exchange splitting of majority and minority subbands. For $T / T_{C}^{B}=0.48$, the energy separation of the two spin components of both bulk feature is approximately $0.27 \mathrm{eV}$. At $\bar{M}$ these bands exhibit a

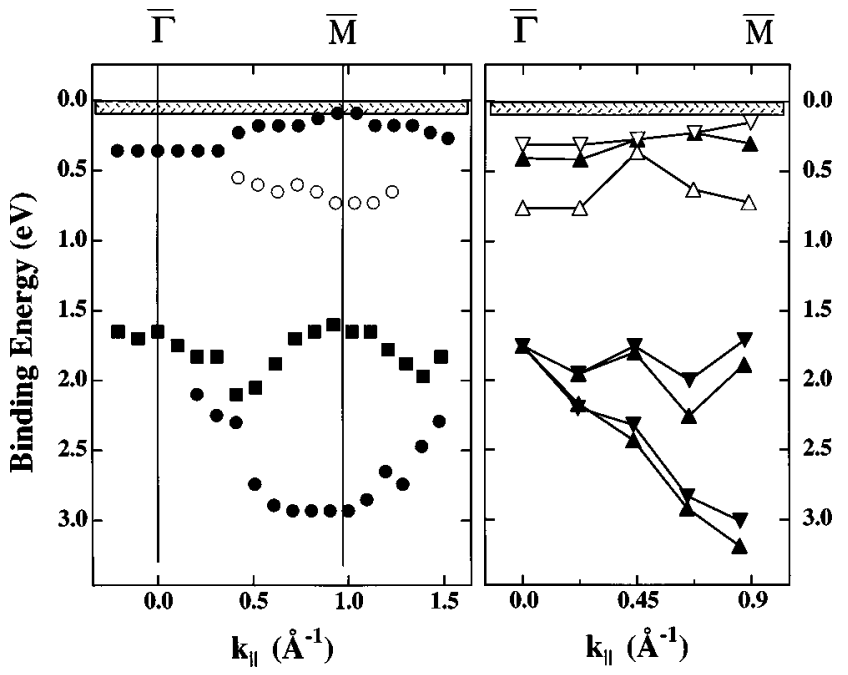

FIG. 5. Spin-integrated (left) and spin-resolved (right) band structure of a 40-ML-thick strained Gd film at approximately 145 $\mathrm{K}$. The filled symbols indicate bands with bulk character while the open symbols mark the binding-energy positions of states with surface character. The spin-resolved band structure in the right panel indicates majority $(\triangle)$ and minority $(\nabla)$ bands. The hatched region near the Fermi level indicates the limited resolution.

"Stoner-like" collapse of the exchange splitting as is evident by the decrease of the exchange splitting with increasing temperature (Fig. 3, left panel). The bulk bands of strained $\mathrm{Gd}(0001)$ at $\bar{M}$ are not purely Stoner-like in behavior, as all four subbands expected with spin-mixing behavior, can be readily identified for the bulk band at $3 \mathrm{eV}$ binding energy for $T / T_{C}^{B}=0.48$, as seen in Fig. 3 .

Further evidence for wave-vector-dependent exchange splitting is provided by emission angle-dependent spinresolved valence-band spectra of a 40-ML-thick strained Gd film as shown in Fig. 4. The majority and minority subbands of the valence band are plotted across the surface Brillouin zone from $\bar{\Gamma}$ to $\bar{M}$. It can be seen that the bulk bands at 1.8 $\mathrm{eV}$ below $E_{F}$ at $\bar{\Gamma}$ disperse and split into two branches. Accompanying the dispersion of these occupied bulk bands of $\Delta_{5}$ or $\Delta_{6}$ symmetry at $\Gamma$ with increasing wave vector, the exchange splitting gradually increases, reflecting the transition from little or no exchange splitting at $\Gamma$ to the large exchange splitting of the strained occupied Gd bulk bands towards $\bar{M}$.

A spin-resolved experimental band structure from $\bar{\Gamma}$ to $\bar{M}$, constructed from the angle-dependent spin-resolved valence-band spectra (Fig. 4), is presented in the right panel of Fig. 5 for $T / T_{C} \approx 0.5$, while the spin-integrated band structure is shown in the left panel of Fig. 5. The spin-resolved band structure confirms the increase in exchange splitting of the Gd $5 d_{x z, y z}, 6 p_{x, y}$, or $5 d_{x^{2}-y^{2}}$ bulk bands with increasing wave vector from less than $0.05 \mathrm{eV}$ at $\Gamma$ to $0.27 \mathrm{eV}$ at $\bar{M}$. The actual change in exchange splitting across the Brillouin zone has been plotted in Fig. 6. The spin-resolved band-structure measurements (Fig. 4) also reveal the existence of three subbands near the Fermi level, which cannot be resolved in the spin-integrated band structure. ${ }^{27}$ The 

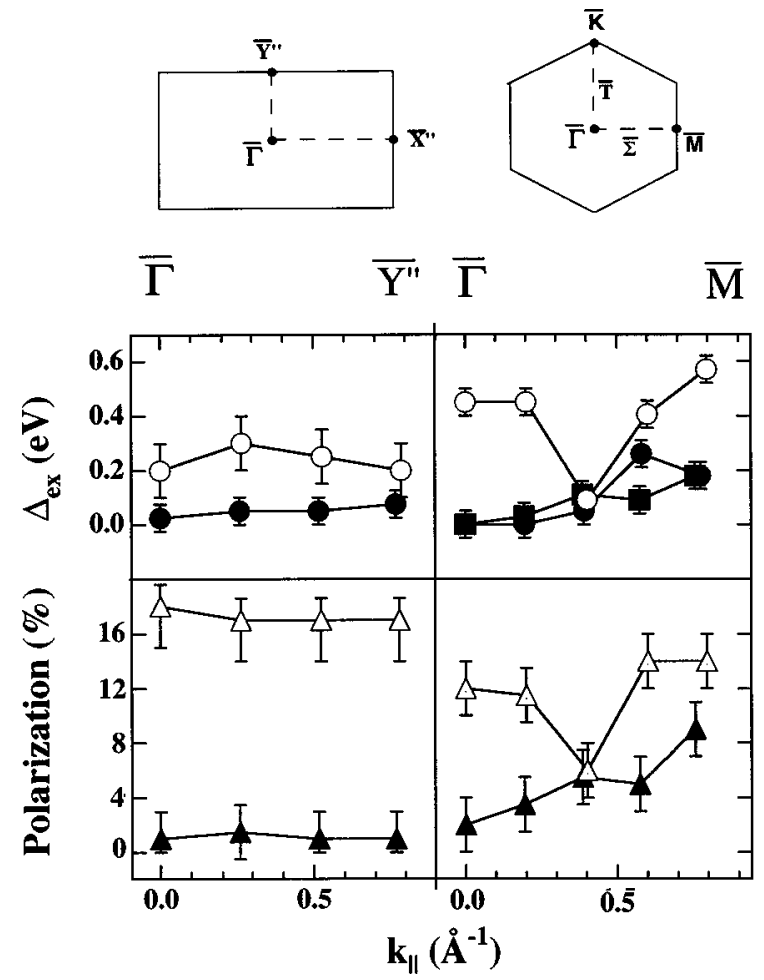

FIG. 6. The exchange splitting (top) and spin asymmetry above background (bottom) of the surface (open symbols) and bulk (filled symbols) spin subbands as a function of wave vector. Data are shown for a strained 4-ML-thick (101̄2) thin film (left) and a 40ML-thick (right) strained (0001) Gd film grown on Mo(112). The data points have been extracted from spin-polarized photoemission spectra at $145 \mathrm{~K}$. The Brillouin-zone symmetry points are indicated at the top.

region of background polarization is, however, greatest about the zone center as seen in Fig. 7.

\section{WAVE-VECTOR DEPENDENCE OF THE SURFACE- STATE EXCHANGE SPLITTING}

At the high symmetry points, the surface character of the spin-majority band at $0.75 \mathrm{eV}$ binding energy and the spinminority band at $0.2 \mathrm{eV}$ binding energy $\left(T / T_{C} \approx 0.5\right)$ is clear

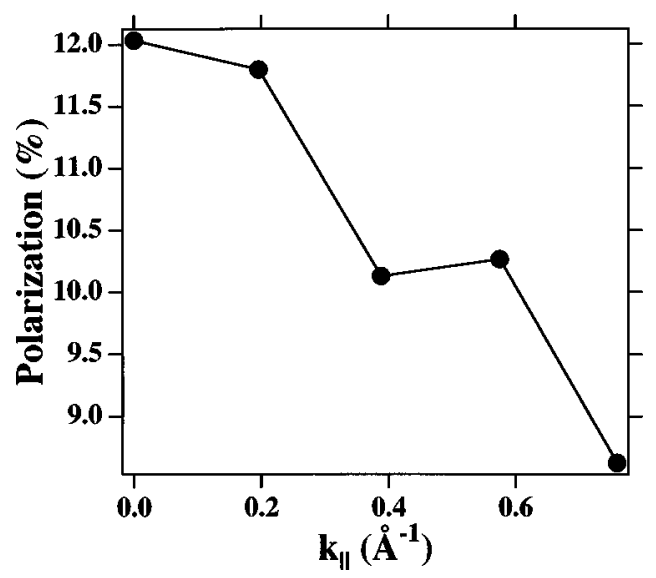

FIG. 7. Wave-vector dependence of the valence background polarization. The data points present the averaged background, integrated over the entire valence-band region.
(Figs. 3 and 4). In the region of the zone about halfway along $\overline{\Gamma \Sigma M}$, the surface spin-minority band overlaps with the bulk spin-majority band (Figs. 4 and 5) and bulk character contributes to the otherwise surface-sensitive band. We have postulated that the lowered symmetry, in midzone, allows hybridization of the surface state with the bulk majority bands near the Fermi level. Due to the $k$-dependent hybridization of the surface and bulk electronic structure, the states with considerable surface sensitivity resemble surface states at both high symmetry points $\bar{\Gamma}$ and $\bar{M}$ but are more like a surface resonance in the Brillouin-zone interior. ${ }^{27}$

The surface states/surface resonances of the strained Gd(0001) disperse less than the bulk bands (Figs. 4 and 5) and are therefore far more localized than the bulk bands, as is the case for unstrained $\operatorname{Gd}(0001) .^{12,14,41}$ The wave-vectordependent exchange splitting of the surface states/resonances is different from the bulk and so is the magnetic behavior. This is evident in the distinct wave-vector dependence of the surface bulk band exchange splittings strained Gd(0001) plotted and compared with the bulk band exchange splitting in Fig. 6. The surface-state exchange splitting is large at the Brillouin-zone center $\left(\Delta_{\mathrm{ex}} \approx 0.45 \mathrm{eV}\right)$ and at the Brillouinzone edge $\left(\Delta_{\mathrm{ex}} \approx 0.57 \mathrm{eV}\right)$, but develops a minimum of the exchange splitting at the Brillouin-zone interior $\left(\Delta_{\mathrm{ex}}\right.$ $\approx 0.10 \mathrm{eV})$. The region in the Brillouin zone along $\overline{\Gamma \Sigma M}$ where the exchange splitting is at minimum coincides with the region where the surface spin-minority band overlaps with the bulk spin-majority band. The postulated hybridization of the surface state with the bulk majority bands near the Fermi level results in greater bulk-like behavior in this region of the Brillouin zone. Due to the $k$-dependent hybridization of the surface and bulk electronic structure, the states with considerable surface sensitivity resemble surface states at both high symmetry points $\bar{\Gamma}$ and $\bar{M}$ but are more like a surface resonance in the Brillouin-zone interior. The collapse in the large exchange splitting of the surface-sensitive feature can be attributed to the wave-vector-dependent change from a surface state (zone center and zone edge) to a surface resonance (zone interior). ${ }^{27}$ The dip of the surface exchange splitting is also reflected in the spin asymmetry, which reduces to $4 \%$ in the Brillouin-zone interior as compared to $10 \%$ at the zone center and $12 \%$ at the zone edge (Fig. 6). Nonetheless, for the surface there is little difference between the exchange splitting at the zone center and at the zone edge.

Model calculations for surface states in a correlated local moment film ${ }^{53}$ have shown that the surface states are expected to be strongest at $\bar{\Gamma}$ and $\bar{M}$ and weakest in zone center (at best they could only be described as one contribution to a bulk band), as is observed in the experimental studies of strained $\operatorname{Gd}(0001)$ described here. These model calculations ${ }^{53}$ have resulted in surface-state band dispersion, relative to a bulk band near the Fermi level, that are very similar to the experimental results shown here. If the model calculations can be compared to the experimental results described here, this suggests that the hopping is either much greater or much weaker in the surface, at the $\bar{\Gamma}$ and $\bar{M}$ points within the Brillouin zone, than is the case for the bulk. 


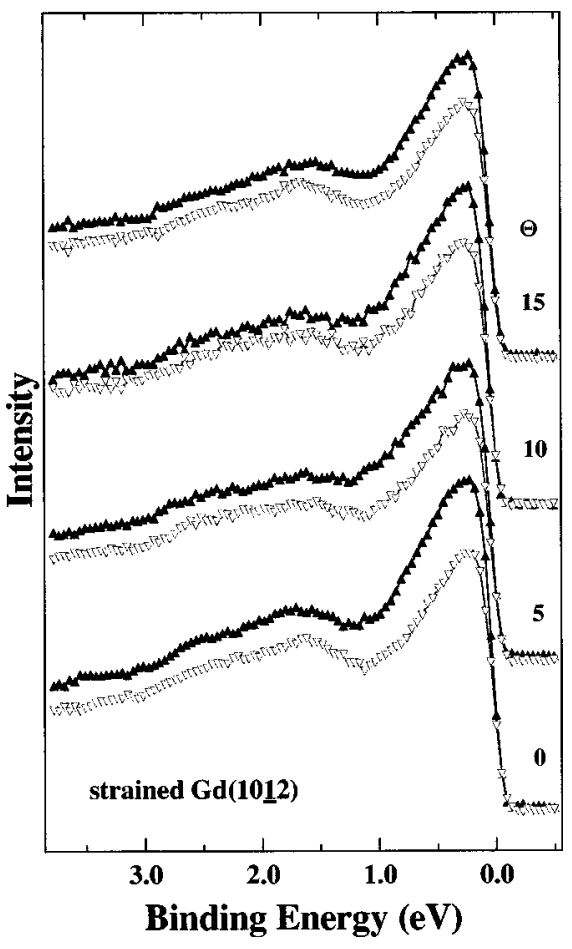

FIG. 8. Emission angle-dependent spin-polarized photoemission spectra of a 4-ML gadolinium film on Mo(112) at $145 \mathrm{~K}$. These spectra of the "strained Gd(1012)" thin film exhibit little bulk band exchange splitting, the features at higher binding energies (see text).

\section{WAVE-VECTOR DEPENDENCE OF ULTRA- THIN GADOLINIUM FILMS GROWN ON Mo(112)}

Thin films of 3-20 ML of gadolinium grown on Mo(112) adopt a structure similar to that of strained $\operatorname{Gd}(10 \overline{12}){ }^{27}$ These thinner films, as yet, cannot be grown with the flat surface and crystalline order of the thicker $40 \mathrm{ML}$ of strained $\mathrm{Gd}(0001)$ on $\mathrm{Mo}(112),{ }^{54}$ but band structure is evident. ${ }^{27}$

The band structure of strained $\operatorname{Gd}(10 \overline{1} 2)$ is distinct from that of strained $\mathrm{Gd}(0001)$, which is manifested in the negligible dispersion of the bulk bands $\left(1-2 \mathrm{eV}\right.$ below $\left.E_{F}\right) .{ }^{27}$ The exchange splitting of the bulk bands well below the Fermi level ${ }^{27}$ of this thinner film (with the rectangular Brillouin indicated in Fig. 6) is small throughout the surface Brillouin zone and exceeds the experimental resolution of $0.05 \mathrm{eV}$ only near the zone edge $\bar{Y}^{\prime \prime}\left(\Delta_{\mathrm{ex}} \approx 0.07 \mathrm{eV}\right)$, as seen in Fig. 8 for a 4-ML-thick film. This is summarized in Fig. 6. There is also no significant polarization (above background) in the region of the Gd bulk bands for any wave vector. The exchange splitting of the surface state of the $\operatorname{Gd}(1012)$ films is of the order of $0.25 \mathrm{eV}$ (for $T / T_{C} \approx 0.8$ ) with little variation for different wave vectors. This exchange splitting of the surface state must be taken as only an estimate in the absence of spin-polarized inverse photoemission and may be greater if the minority component straddles the Fermi level. The spin polarization in the region of the surface-sensitive states approaches approximately 17\% (above background).

At $\bar{\Gamma}$, the exchange splitting and polarization behavior of the occupied bulk bands, away from the Fermi level is similar for both thin, 3-10 ML, the thicker, $40 \mathrm{ML}$, gadolinium films grown on $\mathrm{Mo}(112)$. As we have already noted, at zone center, the exchange splitting of the $5 d_{x z, y z}, 6 p_{x, y}$, or

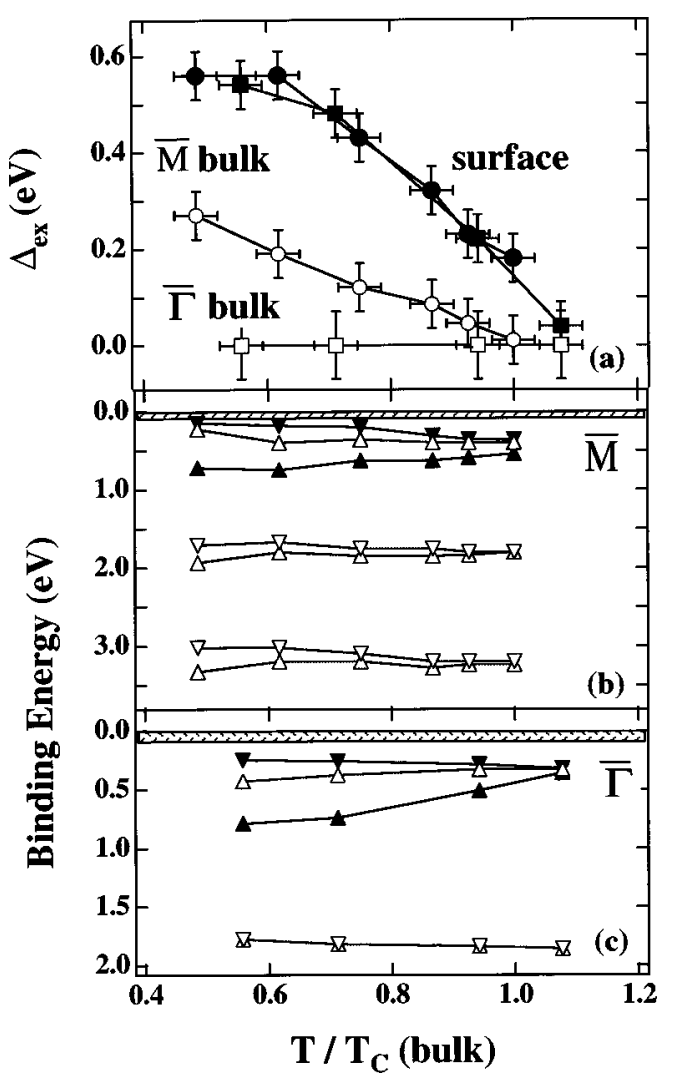

FIG. 9. (a) The temperature-dependent exchange splitting for the surface (filled symbols) and the bulk (open symbols) at $\bar{\Gamma}$ (boxes) and $\bar{M}$ (circles). The temperature-dependent spin-resolved binding energies are displayed in (b) and (c) for wave vectors corresponding to $\bar{M}$ and $\bar{\Gamma}$ of the surface Brillouin zone, respectively. The different symbols indicate majority $(\triangle)$ and minority $(\nabla)$ bands, as well as bands with bulk character (open symbols) and states of surface character (filled symbols).

$5 d_{x^{2}-y^{2}}$ bulk bands is negligible, for either strained $\mathrm{Gd}(0001)$ [40 ML of $\mathrm{Gd}$ on $\mathrm{Mo}(112)]$. This is also seen for strained $\operatorname{Gd}(10 \overline{1} 2)$.

\section{TEMPERATURE DEPENDENCE OF THE EXCHANGE SPLITTING}

The temperature dependence of the occupied spin subbands of strained $\operatorname{Gd}(0001)$, as shown in Fig. 3, is summarized in Fig. 9, excluding the bulk bands close to the Fermi level. Figures 9(b) and 9(c) present the occupied subband binding energies at $\bar{M}$ and $\bar{\Gamma}$, respectively, while Fig. 9(a) summarizes the exchange splitting of surface and bulk occupied bands and these high symmetry points. At $\bar{M}$ the exchange split majority and minority components of both bulk and surface merge nearly symmetrically with increasing temperature. At the bulk Curie temperature $\left[T / T_{C}(\right.$ bulk $)=1$ or about $340 \pm 20 \mathrm{~K}]$ the bulk spin subbands overlap, but the surface majority and minority states are still exchange split by approximately $0.1 \mathrm{eV}$, accompanied by some persistent spin asymmetry in the region of the surface state, indicative of an enhanced surface Curie temperature $(370 \pm 25 \mathrm{~K})$. For the $\bar{\Gamma}$ high symmetry point the surface is characterized by an exchange splitting that decreases with increasing temperature 
unlike the bulk band at $1.8 \mathrm{eV}$ binding energy, which does not exhibit any significant exchange splitting throughout the mapped temperature region. Figure 9(a) displays the temperature-dependent exchange splitting of the $\mathrm{Gd}$ $5 d_{x z, y z}, 6 p_{x, y}$, or $5 d_{x^{2}-y^{2}}$ bulk bands and the $5 d_{z^{2}-r^{2}}$, $6 s$ surface state for two wave vectors corresponding to the high symmetry points $\bar{M}$ and $\bar{\Gamma}$. As we have noted, the bulk bands do not exhibit an observable exchange splitting at $\bar{\Gamma}$ but have substantial temperature-dependent exchange character at $\bar{M}$.

The surface exhibits "Stoner-like" collapsing band behavior with exchange splitting energies about twice as large as compared to the largest bulk exchange splitting $(\bar{M})$. Given the large electron localization indicated by the absence of surface-sensitive band dispersion, we suggest that in addition to the very large exchange splitting of the surface state, the surface electronic structure also exhibits some spinmixing behavior. The data are consistent with this postulate, as indicated by a concomitant loss in spin asymmetry of the spin-majority surface state (Fig. 3) with some indications of all four expected subbands at $\bar{M}$ of both the surface state and the occupied bulk bands at about $3 \mathrm{eV}$ binding energy (Figs. 3 and 4). The large exchange splitting of the surface state (particularly at $\bar{\Gamma}$ ) and Stoner-like exchange splitting collapse do not exclude spin-mixing behavior in the surface state and this may vary with wave vector. An admixture of spin-mixing and Stoner-like magnetism in the surface layer and at least some of the bulk bands is postulated and this admixture is clearest at $\bar{M}$. Such a mixture of spin-mixing behavior and Stoner-like ferromagnetism has been demonstrated for the $\mathrm{Gd}(0001)$ surface state for gadolinium grown on $\mathrm{W}(110)$ (Refs. 12 and 42) and this behavior is also observed in itinerant moment magnetic systems, such as $\mathrm{Fe}^{55}$

\section{LONG-RANGE VERSUS SHORT-RANGE MAGNETIC ORDER}

The wave-vector-dependent exchange splitting is profound for the occupied $\Delta_{5}, \Delta_{6}$ symmetry (or $5 d_{x z, y z}, 6 p_{x, y}$, or $5 d_{x^{2}-y^{2}}$ character) strained Gd bulk bands. The large ferromagnetic exchange splitting of these bulk bands (well below the Fermi level) at the zone edge $(\bar{M})$ reflects the dominant role of short-range magnetic order on these occupied $\Delta_{5}, \Delta_{6}$ symmetry (or $5 d_{x z, y z}, 6 p_{x, y}$, or $5 d_{x^{2}-y^{2}}$ ) bulk bands for strained $\mathrm{Gd}(0001)$. The absence of exchange splitting and polarization, above background for the bulk bands at $1.5-2 \mathrm{eV}$ binding energy for both the strained $\operatorname{Gd}(10 \overline{1} 2)$ thin films and the strained $\mathrm{Gd}(0001)$ thicker films at $\bar{\Gamma}$, results in these bulk bands resembling the background or paramagnetic bands in close contact with a ferromagnet. This behavior of these bulk bands, at zone center, is similar to the polarization of the $\mathrm{Cu} 3 d$ bands observed in the 2-3-ML $\mathrm{Cu} / \mathrm{Co}$ system. ${ }^{56} \mathrm{We}$ suggest that this bulk band at $1.8 \mathrm{eV}$ binding energy has little ferromagnetic character at $\bar{\Gamma}$ in strained $\mathrm{Gd}(0001)$ and reflects that these bulk bands (away from the Fermi level) are insensitive or contribute little to the ferromagnetic long-range order (which corresponds to the zone center of the Brillouin zone of $\bar{\Gamma}$ ). These bulk bands, resembling a polarized paramagnetic band at $\bar{\Gamma}$, gradually transform to ferromagnetic bands at $\bar{M}$ with the characteristic temperature-dependent exchange splitting where the influence of short-range magnetic order on these bulk bands is greatest. This is in contrast to the bulk bands of unstrained Gd(0001), which exhibit significant "Stoner-like" exchange splitting at $\bar{\Gamma} .{ }^{25}$

By comparison, the existence of the occupied spinmajority bulk band state of $\Delta_{1}, \Delta_{2}$ symmetry of $6 s, 6 p_{z}$, or $5 d_{3 r^{2}-z^{2}}$ character close to the Fermi level for strained $\operatorname{Gd}(0001)$, across much of the Brillouin zone, will do much to increase the spin-majority population, at the expense of spin minority. This latter bulk band straddles the Fermi level with the spin-majority weight observed to be, at least largely, occupied, while the spin-minority weight is largely unoccupied at $\bar{\Gamma}$ (Fig. 2). The effect of the increase in the spinmajority density, particularly so close to the Fermi level, may be a significant factor in increasing the Curie temperature of strained gadolinium grown on $\mathrm{Mo}(112)$ as compared to the unstrained gadolinium. The increase in localization must lead to a greater overlap of the $5 d / 6 s$ orbitals with the $4 f$. The concomitant increase in $5 d / 6 s$ polarization more than overcomes the decrease in coupling due to the loss in itinerancy, judging by the increase in the Curie temperature with expansive strain.

From the MOKE hysteresis loop (Fig. 1), strained gadolinium is patently ferromagnetic and this ferromagnetism is reflected in the fact that the bulk bands closer to the Fermi level exhibit a substantial exchange splitting at zone center (Fig. 2). This polarization of the bulk bands near and above the Fermi level also demonstrates that there is, indeed, longrange ferromagnetic order. This long-range magnetic order is also reflected in the fact that the background polarization is greatest at the Brillouin-zone center $(\bar{\Gamma})$, as seen in Fig. 7 . Gadolinium is a local moment system and, unlike $\mathrm{Fe}, \mathrm{Co}$, or $\mathrm{Ni}$, the origin of the large moment in $\mathrm{Gd}$ is the $4 f^{7}$ shallow core level. Coupling occurs through itinerant $5 d, 6 s$ valence electrons, polarized by the $4 f$ moment. From the data presented here, short- and long-range ferromagnetic order does not influence the surface bands and the various bulk bands of strained $\mathrm{Gd}(0001)$ in an identical fashion. The various bands must contribute to the long-range ferromagnetic coupling differently.

There is little difference in the surface-state exchange splitting for strained $\mathrm{Gd}(0001)$ between the Brillouin-zone center and the zone edge. The importance of short-range order may, nonetheless, affect the spin-polarized band structure of the surface state as well. The admixture of spin-mixing behavior and Stoner-like ferromagnetism is most clear at $\bar{M}$. This phenomenon is, perhaps, more expected. The influence of short-range order is anticipated to be greater as one approaches the Curie temperature.

Both the dispersion and the variations in the exchange splitting with wave vector for strained $\mathrm{Gd}(0001)$ on $\mathrm{Mo}(112)$ resemble the spin-polarized band structure of the rare-earth pnictides. ${ }^{21}$ In the rare-earth pnictides, the wave function can shift in weight from pnictide $p$ or $s$ to rare-earth $d$ with increasing wave vector. ${ }^{21}$ This shift in spectral weight with wave vector can alter the exchange splitting because, as has been noted for the gadolinium oxide, ${ }^{57}$ the chalcogen or pnictide atoms in the lattice have less moment. In the el- 
emental local moment system, such a dramatic shift in the wave-function weight is more difficult to accomplish. For strained gadolinium bands of $\Delta_{1}, \Delta_{2}$ symmetry, both $5 d_{z^{2}-r^{2}}$ and $6 s$ rectangular representations contribute but with bands of $\Delta_{5}, \Delta_{6}$, as is the case for the occupied bulk bands at 1.8 $\mathrm{eV}$ binding energy, there is no admixture of the $6 s$ rectangular representation to the band though an influence on wave-vector-dependent exchange splitting from an admixture of $p_{x}, p_{y}$ is possible. It is these latter bands of $\Delta_{5}, \Delta_{6}$ symmetry that exhibit such pronounced wave-vector dependence of the exchange splitting. Thus, regardless of the details of origin, the variation in exchange splitting with wave vector must indeed be an effect of band structure.

\section{CONCLUSIONS}

To our knowledge, the spin-polarized photoemission results from strained thin films of Gd provide the first direct experimental evidence for wave-vector-dependent exchange splitting in a local moment system. This work establishes the principle that electron localization and hybridization with local $4 f$ moments are wave-vector dependent and reflected in the magnetic behavior. The implications are extremely im-

*Author to whom correspondence should be addressed. FAX: 402472-2879. Electronic address: pdowben@unlinfo.unl.edu

${ }^{1}$ R. E. Watson and A. J. Freeman, Phys. Rev. Lett. 14, 695 (1965).

${ }^{2}$ R. E. Watson and A. J. Freeman, Phys. Rev. 152, 566 (1966).

${ }^{3}$ R. E. Watson and A. J. Freeman, Phys. Rev. 178, 725 (1969).

${ }^{4}$ R. E. Watson, A. J. Freeman, and S. Koide, Phys. Rev. 186, 625 (1969).

${ }^{5}$ W. Nolting, T. Dambeck, and G. Borstel, Z. Phys. B 94, 409 (1994); W. Nolting, G. Borstel, T. Dambeck, T. Fauster, and A. Vega, J. Magn. Magn. Mater. 140-144, 55 (1995).

${ }^{6}$ W. Nolting and W. Borgiel, Phys. Rev. B 39, 6962 (1989).

${ }^{7}$ W. Nolting, W. Borgiel, V. Dose, and T. Fauster, Phys. Rev. B 40, 5015 (1989).

${ }^{8}$ J. Hubbard, Phys. Rev. B 23, 5974 (1981).

${ }^{9}$ A. J. Pindor, J. Staunton, G. M. Stocks, and H. Winter, J. Phys. F 13, 979 (1983)

${ }^{10}$ M. Donath, Appl. Phys. A: Solids Surf. 49, 351 (1989); B. Johansson and J. M. Wills, Phys. Rev. B 52, 4420 (1995).

${ }^{11}$ V. Korenman and R. E. Prange, Phys. Rev. Lett. 44, 1291 (1980).

${ }^{12}$ D. Li, J. Zhang, P. A. Dowben, and M. Onellion, Phys. Rev. B 45, 7272 (1992).

${ }^{13}$ D. Li, J. Zhang, R. T. Wu, and M. Onellion, J. Phys.: Condens. Matter 4, 3929 (1992).

${ }^{14}$ Dongqi Li, Jiandi Zhang, P. A. Dowben, and M. Onellion, Phys. Rev. B 48, 5612 (1993).

${ }^{15}$ P. Aebi et al., Phys. Rev. Lett. 76, 1150 (1996).

${ }^{16}$ K.-P. Kämper, W. Schmitt, and G. Güntherodt, Phys. Rev. B 42, 10696 (1990).

${ }^{17}$ A. M. Turner and J. L. Erskine, Phys. Rev. B 28, 5628 (1983).

${ }^{18}$ M. F. Onellion, C. L. Fu, M. A. Thompson, J. L. Erskine, and A. J. Freeman, Phys. Rev. B 33, 7322 (1986).

${ }^{19}$ U. Alkemper et al., Phys. Rev. B 50, 17496 (1994).

${ }^{20}$ M. Getzlaff, J. Bansmann, and G. Schönhense, J. Electron Spectrosc. Relat. Phenom. 77, 197 (1996).

${ }^{21}$ A. G. Petukhov, W. R. L. Lambrecht, and B. Segall, Phys. Rev. B 53, 4324 (1996). portant and fundamental to the understanding of magnetism. Since the Stoner exchange splitting can (in some sense) be related to the correlation energy $U,{ }^{58}$ a more realistic picture of the band structure ought to include wave-vectordependent exchange and correlation energies, i.e., $U$ $=U(k)$. This work represents one of the few (if any) combined spin-polarized photoemission and spin-polarized inverse photoemission studies undertaken on a single system.

As is clear from this work, to fully assess the relationship of electronic structure on the magnetic properties of an elemental system, the combination of spin-polarized photoemission and spin-polarized inverse photoemission is particularly valuable.

\section{ACKNOWLEDGMENTS}

This work was supported by the NSF through Grant Nos. DMR-94-07933, DMR-94-96131, and DMR-98-02126. The experiments were carried out at the National Synchrotron Light Source, which is funded by the DOE. The authors would like to thank R. Schiller, W. Müller, M. Donath, A. Freeman, M. Farle, and D. Singh for their helpful comments.

${ }^{22}$ B. Kim et al., Phys. Rev. Lett. 68, 1931 (1992).

${ }^{23}$ E. Weschke et al., Phys. Rev. Lett. 77, 3415 (1996).

${ }^{24}$ A. V. Fedorov, K. Starke, and G. Kaindl, Phys. Rev. B 50, 2739 (1994).

${ }^{25}$ Dongqi Li, J. Pearson, S. D. Bader, D. N. McIlroy, C. Waldfried, and P. A. Dowben, Phys. Rev. B 51, 13895 (1995).

${ }^{26}$ Dongqi Li, J. Pearson, S. D. Bader, D. N. McIlroy, C. Waldfried, and P. A. Dowben, J. Appl. Phys. 79, 5838 (1996).

${ }^{27}$ C. Waldfried, D. N. McIlroy, and P. A. Dowben, J. Phys.: Condens. Matter 9, 10615 (1997).

${ }^{28}$ B. N. Harmon and A. J. Freeman, Phys. Rev. B 10, 1979 (1974).

${ }^{29}$ B. N. Harmon, U. P. Antropov, A. I. Liechtenstein, J. V. Solovyev, and V. I. Anisinov, J. Phys. Chem. Solids 56, 1521 (1995); O. Eriksson et al., Phys. Rev. B 52, 4420 (1995).

${ }^{30}$ S. Legvold, B. N. Harmon, B. J. Beaudry, P. Bugart, D. R. Younkin, and H. W. White, Phys. Rev. B 16, 4986 (1977).

${ }^{31}$ E. Vescovo et al., Activity Report 1996, Nat. Synch. Light Source, A-25 (1997).

${ }^{32}$ P. D. Johnson et al., Rev. Sci. Instrum. 63, 1902 (1992); J. Unguris, D. T. Pierce, and R. J. Calotta, ibid. 57, 1314 (1986).

${ }^{33}$ F. Ciccaci, H.-J. Drouhin, C. Hermann, R. Houdré, and G. Lampel, Appl. Phys. Lett. 54, 632 (1989).

${ }^{34}$ A. Goldman, V. Dose, and G. Borstel, Phys. Rev. B 32, 1971 (1985).

${ }^{35}$ V. Dose, Th. Fauster, and R. Schneider, Appl. Phys. A: Solids Surf. 40, 203 (1986).

${ }^{36}$ F. Ciccacci, E. Vescovo, G. Chiasia, S. DeRossi, and M. Tosca, Rev. Sci. Instrum. 63, 3333 (1992).

${ }^{37}$ U. Kolac, M. Donath, K. Ertl, H. Liebl, and V. Dose, Rev. Sci. Instrum. 59, 1933 (1988).

${ }^{38}$ W. Grentz, M. Tschundy, B. Reihl, and G. Kaindl, Rev. Sci. Instrum. 61, 2528 (1990).

${ }^{39}$ M. Donath, B. Gubanka, and F. Passek, Phys. Rev. Lett. 77, 5138 (1996).

${ }^{40}$ Dongqi Li, P. A. Dowben, J. E. Ortega, and F. J. Himpsel, Phys. Rev. B 49, 7734 (1994). 
${ }^{41}$ P. A. Dowben, D. N. Mcllroy, and Dongqi Li, in Handbook on the Physics and Chemistry of Rare Earths, edited by K. A. Geschneider and L. Eyring (North-Holland, Amsterdam, 1997), Vol. 24, pp. 1-46.

${ }^{42}$ M. Bode, M. Getzlaff, S. Heinze, R. Pascal, and R. Wiesendanger (unpublished).

${ }^{43}$ R. Wu, C. Li, A. J. Freeman, and C. L. Fu, Phys. Rev. B 44, 9400 (1991).

${ }^{44}$ C. Waldfried, D. N. McIlroy, T. McAvoy, D. Welipitiya, E. Vescovo, and P. A. Dowben, J. Appl. Phys. A: Solids Surf. (to be published); C. Waldfried and P. A. Dowben, in Magnetism and Electronic Correlations in Local Moment Systems, edited by M. Donath, P. A. Dowben, and W. Nolting (World Scientific, Singapore, 1998).

${ }^{45}$ G. Neuhold and K. Horn, Phys. Rev. Lett. 78, 1327 (1997).

${ }^{46}$ N. V. Smith, Phys. Rev. B 32, 3549 (1985); N. V. Smith, N. B. Brooks, Y. Chang, and P. D. Johnson, ibid. 49, 332 (1994); P. Alqvist, Solid State Commun. 31, 1029 (1979); P. M. Echenique and J. B. Pendry, J. Phys. C 11, 2065 (1978).

${ }^{47}$ C. Waldfried, T. McAvoy, D. Welipitiya, P. A. Dowben, and E. Vescovo, Europhys. Lett. 42, 685 (1998).

${ }^{48}$ C. Rau and M. Robert, Phys. Rev. Lett. 58, 2714 (1987); C. Rau and S. Eichner, Phys. Rev. B 34, 6347 (1986); D. Weller, S. F. Alvarado, W. Gudat, K. Schroder, and M. Campagna, Phys. Rev. Lett. 54, 1555 (1985); D. Weller and S. F. Alvarado, Physica B 130, 72 (1985).

${ }^{49}$ H. Tang, T. G. Walker, H. Hopster, D. P. Pappas, D. Weller, and
J. C. Scott, Phys. Rev. B 47, 5047 (1993); H Tang, D. Weller, T. G. Walker, J. C. Scott, C. Chappert, H. Hopster, A. W. Pang, D. S. Dessau, and D. P. Pappas, Phys. Rev. Lett. 71, 444 (1993).

${ }^{50}$ M. Farle, K. Baberschke, U. Setter, A. Aspelmeier, and F. Gerharter, Phys. Rev. B 47, 11571 (1993); G. André, A. Aspelmeier, B. Schulz, M. Farle, and K. Baberschke, Surf. Sci. 326, 275 (1995); A. Aspelmeier, F. Gerhardter, and K. Baberschke, J. Magn. Magn. Mater. 132, 22 (1994).

${ }^{51}$ R. G. Chapman and N. H. March, J. Magn. Magn. Mater. 61, 81 (1986).

${ }^{52}$ H. Bartholin, J. Beille, D. Bloch, P. Boutron, and J. L. Ferou, J. Appl. Phys. 42, 1679 (1971); E. Klocholm, R. J. Gambino, and J. J. Cuomo, in Magnetism and Magnetic Materials 1971, edited by C. D. Graham, Jr., and J. J. Rhyne, AIP Conf. Proc. No. 5 (AIP, New York, 1972), p. 1462.

${ }^{53}$ R. Schiller, W. Müller, and W. Nolting, Eur. J. Phys. B2, 249 (1998).

${ }^{54}$ C. Waldfried, O. Zeybek, T. Bertrams, S. D. Barrett, and P. A. Dowben, Thin Solid Films (to be published).

${ }^{55}$ E. Kisker, M. Schroder, M. Campagna, and W. Gudat, Phys. Rev. Lett. 52, 2285 (1983).

${ }^{56}$ C. Carbone et al., Phys. Rev. Lett. 71, 2805 (1993).

${ }^{57}$ D. N. McIlroy, C. Waldfried, Dongqi Li, J. Pearson, S. Bader, D.-J. Huang, P. D. Johnson, R. F. Sabirianov, S. S. Jaswal, and P. A. Dowben, Phys. Rev. Lett. 76, 2802 (1996).

${ }^{58}$ V. I. Anisomov, J. Zaanen, and O. K. Anderson, Phys. Rev. B 44, 943 (1991). 